\title{
Differential genetic responses to the stress revealed the mutation-order adaptive divergence between two sympatric ginger species
}

Bing-Hong Huang ${ }^{1}$, Yuan-Chien Lin' ${ }^{2}$, Chih-Wei Huang ${ }^{1}$, Hsin-Pei Lu' ${ }^{1}$ Min-Xin Luo ${ }^{1}$ and Pei-Chun Liao ${ }^{1 *}$ (D)

\begin{abstract}
Background: Divergent genetic responses to the same environmental pressures may lead sympatric ecological speciation possible. Such speciation process possibly explains rapid sympatric speciation of island species. Two island endemic ginger species Zingiber kawagoii and Z. shuanglongensis was suggested to be independently originated from inland ancestors, but their island endemism and similar morphologies and habitats lead another hypothesis of in situ ecological speciation. For understanding when and how these two species diverged, intraspecific variation was estimated from three chloroplast DNA fragments (cpDNA) and interspecific genome-wide SNPs and expression differences after saline treatment were examined by transcriptomic analyses.

Results: Extremely low intraspecific genetic variation was estimated by cpDNA sequences in both species: nucleotide diversity $\pi=0.00002$ in Z. kawagoii and no nucleotide substitution but only indels found in $Z$. shuanglongensis. Nonsignificant inter-population genetic differentiation suggests homogenized genetic variation within species. Based on 53,683 SNPs from 13,842 polymorphic transcripts, in which 10,693 SNPs are fixed between species, Z. kawagoii and Z. shuanglongensis were estimated to be diverged since 218 238 thousand generations ago (complete divergence since $41.5 \sim 43.5$ thousand generations ago). This time is more recent than the time of Taiwan Island formation. In addition, high proportion of differential expression genes (DEGs) is non-polymorphic or non-positively selected, suggesting key roles of plastic genetic divergence in broaden the selectability in incipient speciation. While some positive selected DEGs were mainly the biotic and abiotic stress-resistance genes, emphasizing the importance of adaptive divergence of stress-related genes in sympatric ecological speciation. Furthermore, the higher proportional expression of functional classes in Z. kawagoii than in Z. shuanglongensis explains the more widespread distribution of $Z$. kawagoii in Taiwan.
\end{abstract}

Conclusions: Our results contradict the previous hypothesis of independent origination of these two island endemic ginger species from SE China and SW China. Adaptive divergent responses to the stress explain how these gingers maintain genetic differentiation in sympatry. However, the recent speciation and rapid expansion make extremely low intraspecific genetic variation in these two species. This study arise a more probable speciation hypothesis of sympatric speciation within an island via the mutation-order mechanism underlying the same environmental pressure.

Keywords: Adaptive divergence, Divergent time, Ecological speciation, Mutation-order mechanism, Plastic divergence, Positively selected DEGs

\footnotetext{
* Correspondence: pcliao@ntnu.edu.tw

${ }^{1}$ School of Life Science, National Taiwan Normal University, No. 88, Sec. 4,

Ting-Chow Rd., Wenshan Dist, Taipei 11677, Taiwan

Full list of author information is available at the end of the article
}

(c) The Author(s). 2018 Open Access This article is distributed under the terms of the Creative Commons Attribution 4.0 International License (http://creativecommons.org/licenses/by/4.0/), which permits unrestricted use, distribution, and reproduction in any medium, provided you give appropriate credit to the original author(s) and the source, provide a link to the Creative Commons license, and indicate if changes were made. The Creative Commons Public Domain Dedication waiver (http://creativecommons.org/publicdomain/zero/1.0/) applies to the data made available in this article, unless otherwise stated. 


\section{Background}

Positive divergent selection is a key for driving or maintaining the species divergence in sympatry [1]. Particularly for the island species which usually physical contact, endogenous genetic barrier is important to the reproductive isolation between species of incipient speciation [2, 3]. In fact, ecological speciation could be much faster if selective pressures act on stress-related genes (e.g. cold resistance and starvation tolerance in Drosophila melanogaster [4]). Differential responses to environmental pressures reinforce and fasten the reproductive isolation [5-7]. If these differential responses have similar fitness advantages, selection may promote and maintain the incompatibility of genotypic combinations and is against gene flow via the Bateson-Dobzhansky-Muller incompatibility mechanism $[8,9]$. This process particularly commonly used to explain the parapatric speciation of continental-island species with a continental relative [9]. In addition, ragged topography also creates ecological and altitudinal separation between populations/species (e.g. genera Howea, Metrosideros and Coprosma in Lord Howe Island [10-12]) that facilitates the speciation and radiation of island species. No matter parapatic or sympatric speciation of continentalisland species, the natural selection usually involves in during speciation [13-16], despite a certain cases focused on the geographic isolation (allopatric speciation) [17-20].

Several novel species of genus Zingiber Mill. (Zingiberaceae Martinov) in Southeast Asia have been published due to distinguishable floral variation [21-24] while some ambiguous species were revised and combined [25] in recent years, implying some outstanding taxonomic problems in this genus in SE Asia. There are four native Zingiber species in Taiwan, a continental island off the continental Asia with a central mountain range up to near 4000-m height [26]. Among these four island natives, Z. kawagoii Hayata and Z. shuanglongensis C. L. Yeh \& S. W. Chung are endemic to Taiwan and similar in morphology except slight differences of the rhizome color, bract size and color, and labellum color [26]. However, these two island endemic gingers were suggested to be phylogenetically separated by two inland allies $Z$. smilesianum Craib and Z. striolatum Diels that distributed in southwestern China (Fig. 1) [23].

Zingiber oligophyllum, previously reported as endemic to Taiwan, is now recorded for southeastern mainland China and Hong Kong [27]. Therefore, according to Yeh et al.'s [23] phylogenetic inference, Z. kawagoii and Z. shuanglongensis could be independently derived from widespread ancestors (Fig. 1). However, phylogenetic inference based on few genetic markers is easily affected by incomplete lineage sorting, particularly in species of frequent hybridization such as species of Zingiberaceae [28, 29]. In addition, Z. smilesianum and Z. striolatum, the sisters of $Z$. kawagoii, are widespread in SE Asia but not distributed in Taiwan. Therefore, we propose another hypothesis of recent and sympatric speciation for Z. kawagoii and Z. shuanglongensis according to their morphological similarity, island endemism and sympatric distribution (Fig. 1).

However, despite sympatric in Taiwan, some altitudinal differences between $Z$. kawagoii and Z. shuanglongensis are predicted by ecological niche modeling (ENM) based on 19 bio-climatic data from WorldClim database (Fig. 1). This suggests Grinnellian niche differentiation between these two species. According to different hypotheses of species origination, such niche differentiation could be a cause (e.g. sympatric ecological speciation) or a consequence (e.g. different originations or allopatric speciation) of speciation: if the former, we expected to detect genomic signatures of positive selection

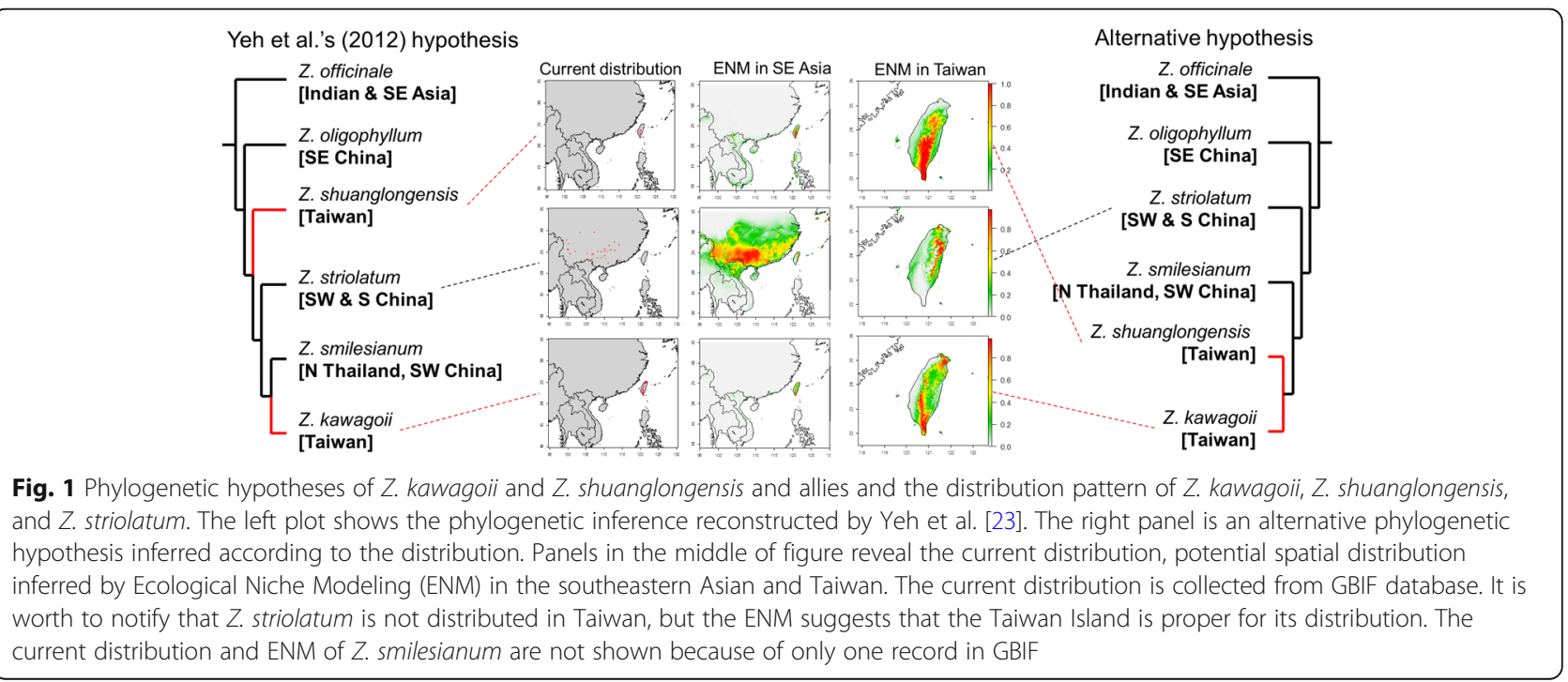


(i.e. adaptive divergence); if the latter, no or few response difference to the environmental pressures is expected.

In fact, the real distributional elevations of these two species are overlap (Z. kawagoii: 300-1500 m; Z. shuanglongensis: 1000-1400 m) with similar microhabitats (under forest, forest edge, slopes, and valley) [26]. This implies these two ginger species suffer similar environmental pressures. Therefore, if there is an adaptive divergence between $Z$. kawagoii and $Z$. shuanglongensis, it should be the mutation-order speciation $[5,30]$, says different mutations fixed in separate populations adapting to similar selective pressure leading divergence, rather than the divergent selection between different environments [5]. Exogenous stress is a major driver leading ecological divergence via selection [2]. Hence the genomic signature of adaptive divergence to the same stress was used for testing the mutation-order mechanism in this study.

Except genetic change, expressional plasticity plays another adaptive component to environmental change [31], by which different species with the same genotype may have differential expressions to response the same stress, i.e. reaction norms [32]. Many researches have indicated the key roles of plastic divergence for the incipient ecological speciation [31, 33, 34]. We tested two contrast hypotheses of adaptive vs. plasticity divergence between two ginger congeners. The genetic response to the stress only different in expression with no signals of positive selection (i.e. no nucleotide difference, selective constraints, and neutrality) is defined as plastic divergence. In contrast, the genes that are differentially expressed and positively selected are defined as adaptive divergence.

In this study, the genome-wide analysis is used to answer question about when and how Z. kawagoii and Z. shuanglongensis divergence. The objectives of this study were twofold: the first, to clarify whether these two island species are recently divergent in Taiwan or independently derived from inland ancestors; second, to test whether and how selective pressure drives or maintains the divergence in incipient speciation. To solve these questions, the chloroplast DNA (cpDNA) sequences were used to evaluate the intraspecific polymorphisms and interspecific divergence, and the genome-wide single nucleotide polymorphisms (SNPs) and RNA expression between these two species were collected via transcriptomic (RNA-seq) technology. Both sampled species were treated with saline before the experiment of RNA-seq in order to understand the genetic response under the stress of osmotic pressure change. Besides, such mild stress helps to increase overall transcription level and is conducive to obtain more loci for inferring the divergence of these two ginger congeners $[35,36]$. The obtained SNPs were collected for divergent time estimation, and missense and silent SNPs were identified to detect signals of positive selection. Cross-reference of differential expression genes (DEGs) with positively selected genes (PSGs) was further used to determine the adaptive and/or plastic divergence between $Z$. kawagoii and Z. shuanglongensis. This study indicates a key role of genome-wide plastic divergence with adaptive divergence in stress-resistance genes between incipient species in sympatry.

\section{Results}

Low intraspecific genetic variation estimated by cpDNA in both species

We adopted three cpDNA region to evaluate the intraspecific divergence before inferring the genome-wide divergence between these two ginger congeners, including $t r n \mathrm{H}-p s b \mathrm{~A}$ intergenic spacer, $r p l 16$ intron, and $\operatorname{trn\mathrm {L}-trn\mathrm {F}}$ intergenic spacer. Total nine $Z$. kawagoii populations and three $Z$. shuanglongensis populations were sampled (Table 1), which cover known distribution of these two species. The concatenated cpDNA sequences revealed very low intraspecific variation in these two species (nucleotide diversity $\pi=0.00002$ in $Z$. kawagoii, while only indels but no SNP were found in $Z$. shuanglongensis). Furthermore, very low interpopulation divergences were observed in these two ginger congeners (Table 2, all corrected intraspecific $\pi_{\mathrm{XY}}<0.001$, all intraspecific $F_{\mathrm{ST}}$, the index of population differentiation, were nonsignificant). These results implied very little intraspecific variation, and the low intraspecific variation might be critical during inferring the divergence between species [37].

\section{Species divergence between two ginger congeners estimated from transcriptomes}

A total of 14.59 and 14.70 million paired-end high-quality reads, in which 2.189 and 2.206 billion bases, with sequence error rate $<1 \%$ (Q20 percentages) of $96.36 \%$ and

Table 1 Sampled population in chloroplast sequence amplification

\begin{tabular}{lllll}
\hline population code & location & sample size & latitude & longitude \\
\hline Z. kawagoii & & & & \\
ZK_DSS & Dahsushan & 8 & N24.233 & E120.900 \\
ZK_WLS & Weiliaoshan & 7 & N22.879 & E120.646 \\
ZK_WLFS & Wulai, Fushan & 9 & N24.777 & E121.504 \\
ZK_RS & Rueishuei & 9 & N23.511 & E121.330 \\
ZK_NZ & Nanzhuang & 10 & N24.574 & E121.044 \\
ZK_KTS & Kantoushan & 6 & N23.267 & E120.501 \\
ZK_JIL & Jiajinlin & 8 & N22.402 & E120.840 \\
ZK_GK & Gukeng & 10 & N23.631 & E120.617 \\
ZK_DTDL & Datong Dali Trail & 12 & N24.192 & E121.637 \\
Z. shuanglongensis & Dulanshan & 12 & N22.894 & E121.186 \\
ZS_DLS & Erjituan & 10 & N23.064 & E120.717 \\
ZS_EJT & Lijia Forest Trail & 10 & N22.805 & E121.032 \\
ZS_LJ & & & & \\
\hline
\end{tabular}


Table 2 Pairwise $F_{S T}$ for population for population divergence in Z. kawagoii (ZK) and Z. shuanglongensis. The corrected average interpopulation difference $\left(\pi_{X Y}\right)$ were shown above the diagonal, while the pairwise $F_{S T}$ were shown below the diagonal

\begin{tabular}{lllllllllllll}
\hline & ZK_DDS & ZK_WLS & ZK_WLFS & ZK_RS & ZK_NZ & ZK_KTS & ZK_JIL & ZK_GK & ZK_DTDL & ZS_DLS & ZS_EJT & ZS_LJ \\
\hline ZK_DDS & - & 0.000 & 0.000 & 0.000 & 0.000 & 0.000 & 0.000 & 0.000 & 0.000 & $22.171^{*}$ & $22.171^{*}$ & $22.171^{*}$ \\
ZK_WLS & 0.037 & - & 0.000 & 0.000 & 0.000 & 0.000 & 0.000 & 0.000 & 0.000 & $22.163^{*}$ & $22.163^{*}$ & $22.163^{*}$ \\
ZK_WLFS & 0.024 & 0.002 & - & 0.000 & 0.000 & 0.000 & 0.000 & 0.000 & 0.000 & $22.163^{*}$ & $22.163^{*}$ & $22.163^{*}$ \\
ZK_RS & 0.000 & 0.038 & 0.025 & - & 0.000 & 0.000 & 0.000 & 0.000 & 0.000 & $22.152^{*}$ & $22.152^{*}$ & $22.152^{*}$ \\
ZK_NZ & 0.000 & 0.069 & 0.059 & 0.000 & - & 0.000 & 0.000 & 0.000 & 0.000 & $22.161^{*}$ & $22.161^{*}$ & $22.161^{*}$ \\
ZK_KTS & 0.000 & 0.017 & 0.000 & 0.000 & 0.000 & - & 0.000 & 0.000 & 0.000 & $22.161^{*}$ & $22.161^{*}$ & $22.161^{*}$ \\
ZK_JIL & 0.000 & 0.026 & 0.011 & 0.000 & 0.000 & 0.000 & - & 0.000 & 0.000 & $22.171^{*}$ & $22.171^{*}$ & $22.171^{*}$ \\
ZK_GK & 0.000 & 0.026 & 0.011 & 0.000 & 0.000 & 0.000 & 0.000 & - & 0.000 & $22.16^{*}$ & $22.16^{*}$ & $22.16^{*}$ \\
ZK_DTDL & 0.000 & 0.051 & 0.040 & 0.000 & 0.000 & 0.000 & 0.000 & 0.000 & - & $22.161^{*}$ & $22.161^{*}$ & $22.161^{*}$ \\
ZS_DLS & $1.000^{*}$ & $0.996^{*}$ & $0.996^{*}$ & $1.000^{*}$ & $1.000^{*}$ & $1.000^{*}$ & $1.000^{*}$ & $1.000^{*}$ & $1.000^{*}$ & - & 0.000 & 0.000 \\
ZS_EJT & $1.000^{*}$ & $0.996^{*}$ & $0.996^{*}$ & $1.000^{*}$ & $1.000^{*}$ & $1.000^{*}$ & $1.000^{*}$ & $1.000^{*}$ & $1.000^{*}$ & 0.000 & - & 0.000 \\
ZS_LJ & $1.000^{*}$ & $0.996^{*}$ & $0.996^{*}$ & $1.000^{*}$ & $1.000^{*}$ & $1.000^{*}$ & $1.000^{*}$ & $1.000^{*}$ & $1.000^{*}$ & 0.000 & 0.000 & - \\
\hline
\end{tabular}

*Significance of random allelic permutation $(P<0.05)$

96.68\% were generated from transcriptomes of Z. kawagoii and Z. shuanglongensis, respectively. Totally 255,711 non-redundant transcripts with N50 value of 1378 bp were generated by Trinity de novo assembly. For pseudoreference-based assembly, contigs of both Z. kawagoii and $Z$. shuanglongensis were assembled together by bowtie2 [38], which results in 205,168 reference sequences $(129,806$ and 138,497 transcripts of Z. kawagoii and Z. shuanglongensis aligned to pseudo-reference, respectively). The mean depth of coverage is $36.426( \pm 147.733$, Additional file 1: Figure S1), and the mean mapping quality is 23.98 .

To estimate species divergent time between $Z$. kawagoii and Z. shuanglongensis, 53,686 SNPs were identified in $13,842(17,302,281$ nucleotides $)$ out of 205,168 transcripts (total length 137,601,681 nucleotides, average length 670.68 nucleotides per transcript), in which 91 transcripts are multi-allelic loci. The variant rate is $3.10 \times 10^{-3}$ per site. Among these SNPs, 10,693 SNPs are fixed differences (fixation rate is $6.18 \times 10^{-4}$ per site). If assuming a constant mutation rate, the divergent time $T$ can be estimated by the formula $\mu=K / 2 T$, where $\mu$ is the mutation rate and the $K$ is the average variants per site or the average fixed differences per site. If applying the mutation rate $6.5 \times 10^{-9}$ or $7.1 \times 10^{-9}$ substitutions/site/generation estimated from Arabidopsis thaliana [39], the time to the most recent common ancestor (TMRCA) of both Z. kawagoii and Z. shuanglongensis could be coalesced back to roughly $2.38 \times 10^{5} \sim 2.18 \times 10^{5}$ generations ago when using variant rate $3.10 \times 10^{-3}$ per site as the $K$. If using the fixation rate $\left(6.18 \times 10^{-4}\right.$ per site) as $K$ to estimate the divergent time, the species divergence was estimated to be $4.75 \times 10^{4} \sim 4.35 \times 10^{4}$ generations ago, which was suggested as the time of completion of speciation.
The plastic species divergence inferred from high proportion of non-positively selected differential expression transcripts

Among these SNPs, 33,571 SNPs locate in exons (56.404\%), 16,170 SNPs in intergenic regions (27.168\%), 4 SNPs in the splice site region $(0.007 \%)$, and 5466 (9.184\%) and 4308 SNPs $(7.238 \%)$ are found in the 5' and $3^{\prime}$ untranslated region (UTR), respectively. Among 33,571 exonic SNPs, there are 14,967 missense variants (44.58\%) and 18,393 synonymous variants (54.79\%), and the other 213 SNPs are initiator codon variants, start lost, stop gained/lost, and stop retained variants $(0.63 \%)$. Slightly smaller proportion of missense variants than the silent variants $(44.58 \%$ vs. $54.79 \%)$ suggests that the overall genomic divergence of these two species is not far from neutral or nearly neutral evolutionary model [40]. The overall distributions of $K a$ and $K s$ values of orthologs are shown in Fig. 2.

We checked the differentially expressed genes using a criterion $\log _{2}$ fold-change $\left(\log _{2} \mathrm{FC}\right)>1$ and $P<0.05$, resulting in 14,402 of 205,168 transcripts (7.02\%) are differentially expressed. In these 14,402 DEGs, 3505 (24.34\%) and 4396 transcripts (35.52\%) are unique to Z. shuanglongensis and Z. kawagoii, respectively; while 6501 DEGs (45.14\%) are commonly expressed in both species, in which 3176 (22.05\%) and 3325 DEGs (23.09\%) are higher expressed in $Z$. shuanglongensis and Z. kawagoii, respectively (Fig. 3). These differential expression patterns not only reflect the congenital differences in gene expression between species but also indicate divergent physiological responses to the saline stress.

Based on the Gene Ontology (GO) annotation, 25,710 (50.00\%), 14,296 (27.80\%), and 11,184 (21.75\%) unigenes of $Z$. shuanglongensis are identified as categories Biological Process (BP), Cell Component (CC), and 


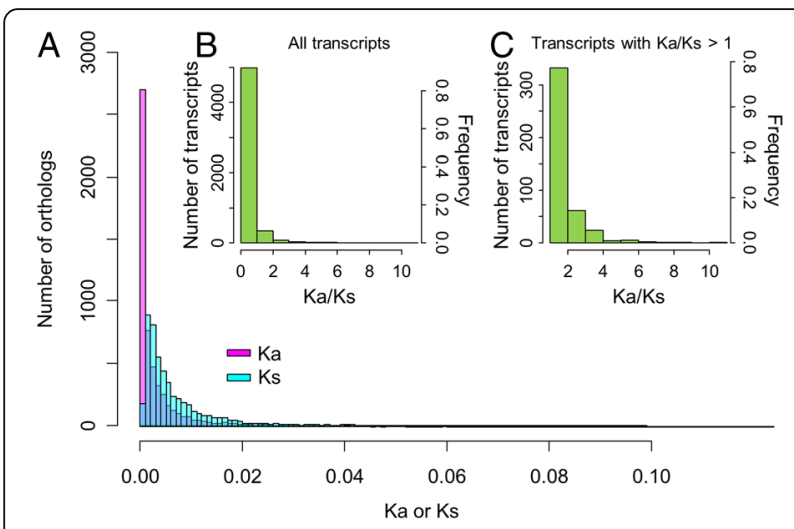

Fig. 2 Distribution of the $K a, K s$, and $K a / K s$ ratio of ortholog pairs under 60 -min saline treatment. (a) Distribution of $K a$ and $K s$ values of all orthologous unigenes reveals a general patten of higher $K s$ than $\mathrm{Ka}$; (b) L-shape Ka/Ks distribution in all transcripts revealed that most homologous genes (96.9\% transcripts) of two ginger species are conserved $(K a / K s<1)$; (c) L-shape distribution of genes with $K a /$ Ks $>1$ indicate that most of the positively selective pressures for the species divergence are slight $(1<K a / K s<2)$

Molecular Function (MF), respectively; 33,689 (50.00\%), 15,295 (22.70\%), and 28,473 (25.66\%) unigenes are assigned as BP, CC, and MF, respectively, in Z. kawagoii. Comparisons of each functional class revealed higher proportion of unique expression of functional classes in $Z$. kawagoii than in $Z$. shuanglongensis, while the commonly expressed functional classes revealed higher expression level in Z. shuanglongensis than in Z. kawagoii (Fig. 4, the GO terms with differences $>0.5 \%$ are shown only). Such differences of expression patterns suggest that, in response to the stress, $Z$. kawagoii may quickly and widely trigger the expression of multiple functional genes (particularly the functional classes in $\mathrm{BP}$ and MF) in contrast to the concentrative expression of part of main functional categories in $Z$. shuanglongensis. The quick response and expression of diversified genes in the face of stresses may be advantageous to adapt changeable environment (i.e. adaptability) and increase the success of colonization (i.e. dispersability) $[41,42]$, which explains the broader habitat ranges of $Z$. kawagoii than $Z$. shuanglongensis in Taiwan (see the current distribution in Fig. 1).

For testing whether such divergence is adaptive or just a phenomenon of plasticity, we further check the $K a / K s$ ratio, a genetic signature of selection [43], of these DEGs. Among 5413 transcripts with exonic SNPs, small proportion of DEGs was found in transcripts with Ka/Ks $>1$ (429/5413 = 7.93\%, Fig. 5). In contrast, relatively high proportion of DEGs was negatively selected or under selective constraint (i.e. $K a / K s<1,4684 / 5413=86.53 \%$, Fig. 5). The result of high proportion of DEGs without under positive selection supports the plasticity hypothesis rather the adaptive hypothesis of genomic divergence between two ginger congeners. Within these positively selected DEGs inferred by $\log _{2} \mathrm{FC}>1$, only 56 DEGs were also significant differences in Fragment Per Kilobase of transcript per Million mapped reads $($ FPKM $)(P<0.05$, Fig. 6$)$, in which 15 DEGs were highly adaptive $(K a / K s>2$, Table 3$)$. These highly adaptive DEGs can be considered to be the key genetic agents that cause or maintain the differentiation of the two ginger congeners.

\section{The adaptive divergence between species inferred from positive selection on stress-resistance DEGs}

We further checked the functions of these highly adaptive-divergent (i.e. $\mathrm{Ka} / \mathrm{K} s>2$ ) DEGs. Ten of 15 transcripts were annotated (Table 4). Five of ten annotated transcripts are disease resistance genes analogous to RGA1 (DN30898_c1_g1_i2), RGA2 (DN32090_c10_g5_i9, DN31496_c1_g1_i13), RPM1 (DN32036_c1_g2_i5), and RPP13-like protein 1 (DN32090_c10_g5_i10); one is analogous to Mitogen-activated protein kinase kinase kinase 1 (MEKK1) (DN26862_c0_g2_i1), with a function of signaling pathway to modulate gene expressions responding to biotic (pathogen defense by negatively regulating innate immunity) and abiotic stresses (cold and salinity stress-mediated MAP kinase signaling cascade); one is analogous to Peroxiredoxin-2C (DN26431_c0_g1_i1), playing a role in cell protection against oxidative stress; and one is an analog of Retrovirus-related Pol polyprotein from transposon TNT 1-94 which might transcribe in the face of stresses (DN28640_c0_g2_i1). These eight transcripts are related to the resistance of biotic and abiotic stresses. Positive selection on these stress-resistance DEGs indicated differential genetic responses to the stress may play important roles in driving or maintaining species divergence.

In addition to the transcript DN28640_c0_g2_i1 analogous to Retrovirus-related Pol polyprotein from transposon TNT 1-94, DN31659_c3_g1_i5 was also annotated as encoding a Retrotransposon gag protein (Table 4). Transcription of TE is known to increase under various kinds of stresses in several systems [44-46]. For example, in Arabidopsis [47] and coral Acropora hyacinthus [48], transposons and retrotransposons are activated during heat stress and are regulated by small interfering RNAs (siRNAs); Copia retrotransposons of the Cucumis melo genome are transcriptionally induced when melon leaves are exposed to UV light [49]; differentially expression of multiple retrovirus-related Pol polyproteins between two bryophytes with divergent desiccation tolerance during cycles of rapid desiccation and rehydration in the intertidal zone differentiates their ecological niches [50]. These two transposons and retrotransposons expressed higher in $Z$. kawagoii than in Z. shuanglongensis (Table 4). Another abiotic stress resistance transcript DN26431_c0_g1_i1 (analog of Peroxiredoxin-2C) is also higher expressed in 


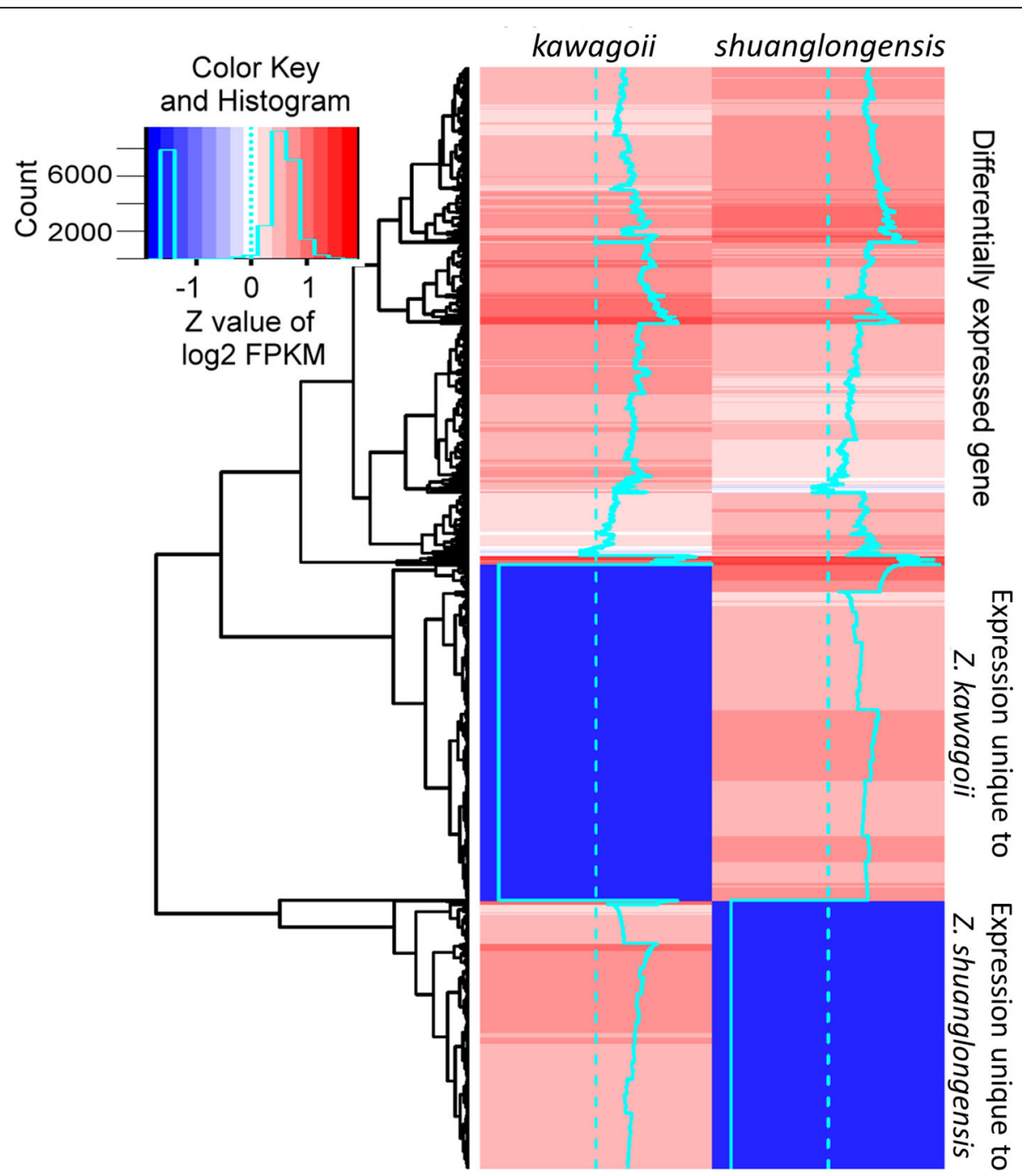

Fig. 3 Comparison of relative expression (FPKM) of differentially expressed genes (DEGs) in heatmap. Only the DEGs fit criteria of log 2 FC $>1$ and significant differences in FPKM $(P<0.05)$ are shown. Three major clusters of transcripts are the genes expressed in both species but differentially expressed and the genes expressed in single species only

Z. kawagoii (Table 4). The high expression of abiotic resistance genes in $Z$. kawagoii may be beneficial to the adaptation to a variety of environments, which explains the wider distribution of Z. kawagoii than Z. shuanglongensis in Taiwan.

The other two transcripts are analogous to the Alpha-humulene 10-hydroxylase (DN31888_c2_g1_i14) which is a catalytic enzyme of zerumbone biosynthesis with an anticancer function, and the Wall-associated receptor kinase 2 (DN31537_c1_g4_i6) functioning in the control of cell expansion, morphogenesis and development (Table 4). The positive selection and differential expression on these genes suggest differential morphogenesis and physiological functions between $Z$. kawagoii and $Z$. shuanglongensis. Ginger ( $Z$. officinalis Rosc.) and other zingiberaceous plants have potent antioxidant and anti-inflammatory activities and are therefore suggested to exhibit cancer preventive activity [51-53]. Differential expression and positive selection on Alpha-humulene 10-hydroxylase analog (DN31888_c2_g1_i14) suggest that Z. shuanglongensis, which has higher expression level, has a higher anti-inflammatory or anticancer effect than Z. kawagoii, although more researches are necessary.

\section{Discussion}

The species divergence event was later than the island formation time

The divergence of these two ginger congeners was since $2.38 \times 10^{5} \sim 2.18 \times 10^{5}$ generations ago and completed at $4.75 \times 10^{4} \sim 4.35 \times 10^{4}$ generations ago. Since the variant rate could be overestimated due to post-speciation 

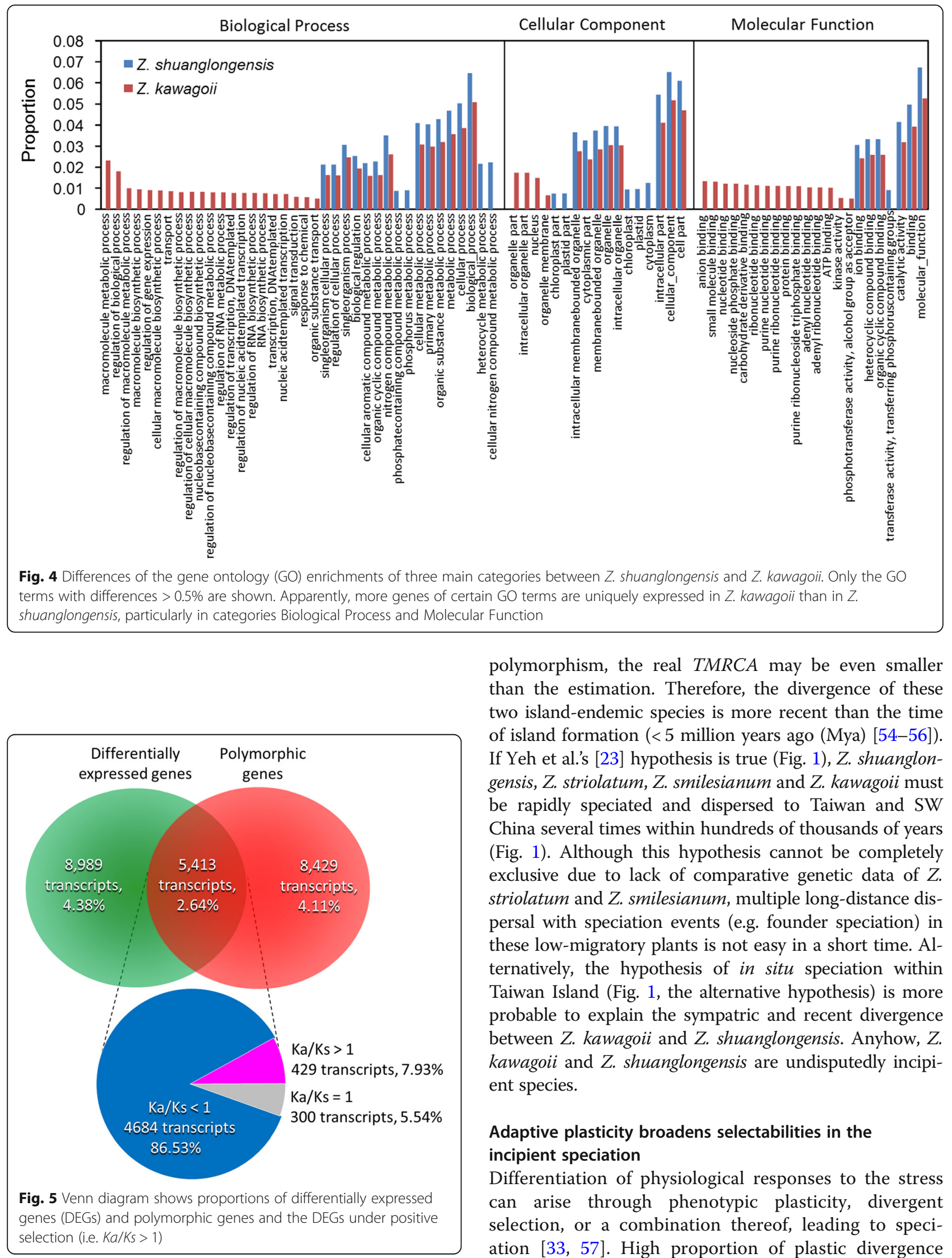

polymorphism, the real TMRCA may be even smaller than the estimation. Therefore, the divergence of these two island-endemic species is more recent than the time of island formation (<5 million years ago (Mya) [54-56]). If Yeh et al.'s [23] hypothesis is true (Fig. 1), Z. shuanglongensis, Z. striolatum, Z. smilesianum and Z. kawagoii must be rapidly speciated and dispersed to Taiwan and SW China several times within hundreds of thousands of years (Fig. 1). Although this hypothesis cannot be completely exclusive due to lack of comparative genetic data of $Z$. striolatum and $Z$. smilesianum, multiple long-distance dispersal with speciation events (e.g. founder speciation) in these low-migratory plants is not easy in a short time. Alternatively, the hypothesis of in situ speciation within Taiwan Island (Fig. 1, the alternative hypothesis) is more probable to explain the sympatric and recent divergence between $Z$. kawagoii and Z. shuanglongensis. Anyhow, Z. kawagoii and $Z$. shuanglongensis are undisputedly incipient species.

\section{Adaptive plasticity broadens selectabilities in the incipient speciation}

Differentiation of physiological responses to the stress can arise through phenotypic plasticity, divergent selection, or a combination thereof, leading to speciation [33, 57]. High proportion of plastic divergence 


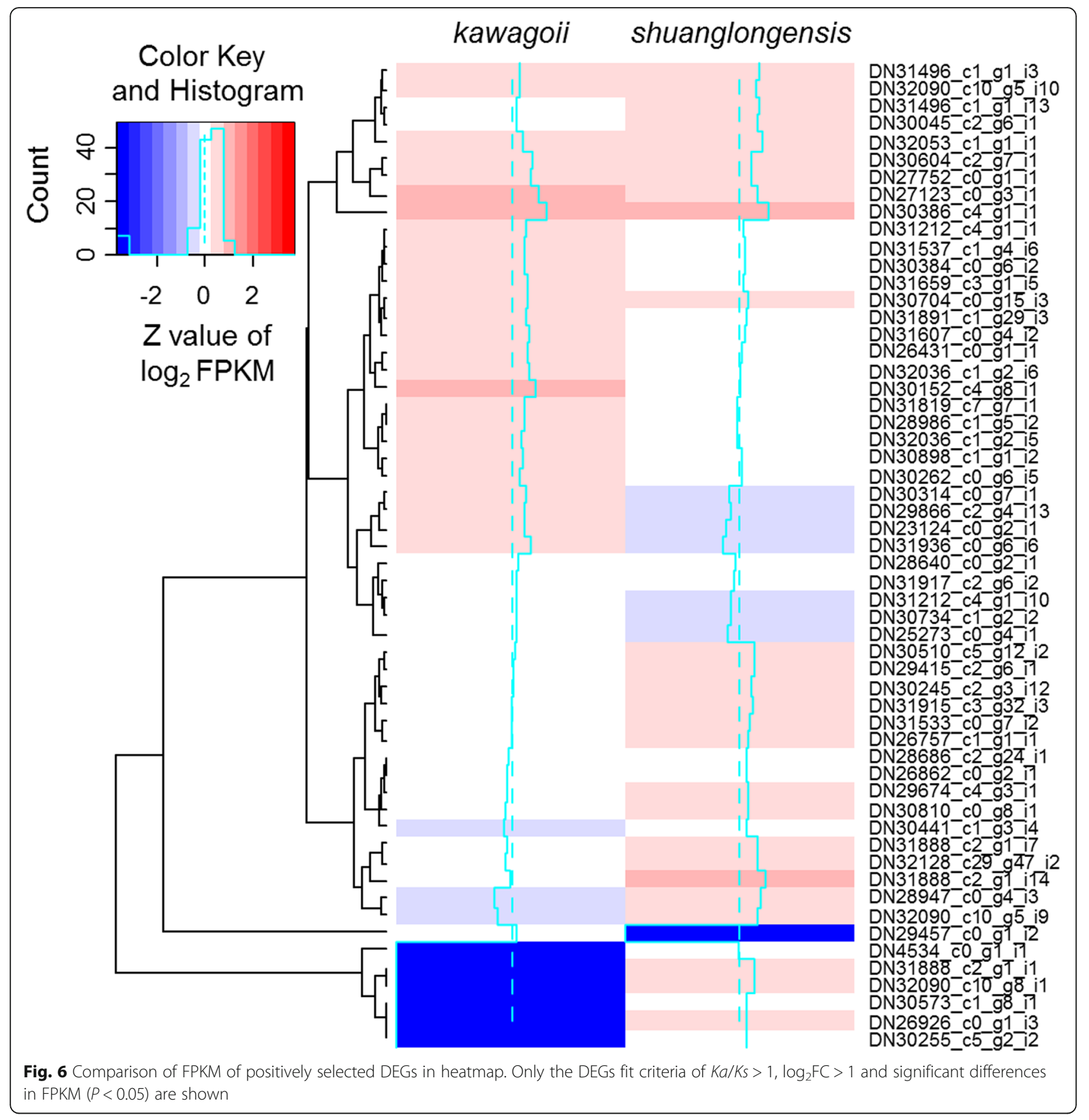

(i.e. DEGs without signatures of positive selection) suggests rapid and pluralistic responses to environmental stresses (e.g. osmotic pressure change due to saline or drought) [58], which increases the variability and selectivity at the beginning of speciation [31]. Adaptive plasticity also facilitates the success of organism to colonize to new habitats, that potentially contributes to genetic differentiation and speciation [59]. The recent divergence between $Z$. shuanglongensis and Z. kawagoii since Middle-to-Late Pleistocene suggests that the speciation initiates in an era of rapid change of climate and environment. High proportion of plastic divergent DEGs facilitates the survival of the common ancestors of $Z$. shuanglongensis and $Z$. kawagoii. If the divergent genetic responses are equally advantageous to the environmental pressure, these two incipient species can persist [8]. Such plasticity extends the persistence of the tolerance of environmental change of populations in low genetic variability, which is common in island endemic species, and the following adaptive evolution can facilitate the persistent responses to environmental change $[58,60]$. 
Table 3 List of SNPs and relative expressions of transcripts with $K a / K s>2$ and significantly differential expression

\begin{tabular}{|c|c|c|c|c|c|c|c|c|}
\hline \multirow[b]{2}{*}{ Transcript Id } & \multirow[b]{2}{*}{ Length } & \multicolumn{3}{|l|}{ SNPS } & \multicolumn{4}{|l|}{ FPKM } \\
\hline & & Missense & Synonymous & $\mathrm{Ka} / \mathrm{Ks}$ & Z. kawagoii (zk) & Z. shuanglongensis (zs) & $\log _{2} F C(z s-z k)$ & $p(z s-z k)$ \\
\hline DN30045_c2_g6_i1 & 386 & 6 & 1 & 3 & 5.12 & 26.67 & 2.29 & 0.038 \\
\hline DN32090_c10_g8_i1 & 634 & 5 & 1 & 2.5 & 0 & 17.47 & 7.97 & $1.80 \mathrm{E}-05$ \\
\hline DN32090_c10_g5_i9 & 722 & 5 & 1 & 2.5 & 0.44 & 27.29 & 5.67 & $1.09 \mathrm{E}-06$ \\
\hline DN31496_c1_g1_i13 & 839 & 16 & 2 & 4 & 5.62 & 31.54 & 2.36 & 0.002 \\
\hline DN32036_c1_g2_i5 & 1148 & 13 & 2 & 3.3 & 9.84 & 1.85 & -2.61 & 0.002 \\
\hline DN31537_c1_g4_i6 & 1778 & 12 & 2 & 3 & 14.86 & 4.92 & -1.77 & 0.016 \\
\hline DN31659_c3_g1_i5 & 2061 & 6 & 1 & 3 & 20.03 & 4.67 & -2.27 & 0.002 \\
\hline DN28640_c0_g2_i1 & 2844 & 6 & 1 & 3 & 5.84 & 1.72 & -1.91 & 0.013 \\
\hline DN30152_c4_g8_i1 & 403 & 5 & 1 & 2.5 & 55.55 & 2.79 & -4.29 & $1.13 \mathrm{E}-04$ \\
\hline DN26431_c0_g1_i1 & 1066 & 6 & 1 & 3 & 22.23 & 3.39 & -2.85 & 3.77E-04 \\
\hline DN30898_c1_g1_i2 & 1872 & 5 & 1 & 2.5 & 11.4 & 3.66 & -1.8 & 0.015 \\
\hline DN32090_c10_g5_i10 & 1862 & 46 & 7 & 3.3 & 7.98 & 24.08 & 1.44 & 0.039 \\
\hline DN31607_c0_g4_i2 & 2162 & 11 & 2 & 2.8 & 27.88 & 5.09 & -2.6 & 3.37E-04 \\
\hline DN31888_c2_g1_i14 & 465 & 7 & 1 & 3.5 & 2.15 & 83.59 & 5.16 & $5.25 \mathrm{E}-07$ \\
\hline DN26862_c0_g2_i1 & 1481 & 7 & 1 & 3.5 & 1.62 & 6.52 & 1.85 & 0.032 \\
\hline
\end{tabular}

Certain nonsynonymous polymorphisms of DEGs are found to be fixed between $Z$. shuanglongensis and $Z$. kawagoii. Such divergent process is particularly revealed in pathogenic and stress-resistance genes (Table 4). In the study of Brassica rapa, pathogenic sustainability changes accompanying with the evolutionary shift of the drought response and even results in early flowering following the drought stress [61]. Although no obvious difference in flowering time between $Z$. shuanglongensis and Z. kawagoii, Z. kawagoii have unique expression of genes regarding the regulation of red or far-red light signaling pathway (GO:0090227). Within this GO term, the transcript DN28195_c2_g11_i1 (analog to the REPRESSOR OF $U V-B$ PHOTOMORPHOGENESIS 2 of Arabidopsis thaliana) that involves in the regulation of photoperiodic flowering and vegetative development have significant higher expression in $Z$. kawagoii than in $Z$. shuanglongensis $\left(\log _{2} \mathrm{FC}=1.866, \quad P=0.009\right)$, and the transcript DN29957_c0_g6_i13 (analog to the Myb transcription factor EARLY FLOWERING MYB PROTEIN, of $A$. thaliana) that acts as a flowering repressor is positively divergent between $Z$. shuanglongensis and $Z$. kawagoii $(K a / K s=1.5)$. Although lack direct evidences to link the expressional differences regarding the pathogenic resistance genes with the flowering regulation under the drought stress, this transcriptomic data revealed clues that the expressional plasticity of stress- and pathogenresistance genes might be associated with the evolution of a reproductive barrier toward adaptive divergence and ecological speciation [33].
Positive selection on stress-resistance genes as an important rule underlying the maintenance of species divergence

Higher expression of genes related to responses to stress and pathogens reveals higher adaptability in Z. kawagoii than in Z. shuanglongensis. Studies on wild sunflower have shown that the positive selection for salt tolerance quantitative trait loci (QTLs) is strong enough to counteract the homogenizing effect of gene flow and facilitate the sympatric speciation $[62,63]$. Divergent responses to the stresses (e.g. saline, pathogens, etc.) accompanying with the gamete incompatibility that results in reproductive barrier during incipient speciation is probably a pleiotropic effect of the evolution of stress- or pathoge n-resistance genes, and the pleiotropic effect may lead to gamete defect and further support the scenario of Bateson-Dobzhansky-Muller incompatibility [3, 8, 9]. The island endemic with overlapping distributions of recently divergent $Z$. kawagoii and Z. shuanglongensis implies the sympatric speciation. Although the speciation drivers and speciation genes are unknown yet, the transcriptomic data revealed that positively divergent selection on stress- and pathogen-resistance DEGs is involved in blocking the genetic homogenization of gene flow between species. These stress- and pathogen-resis tance DEGs are undoubtedly one of the important factors to maintain reproductive isolation in the incipient speciation of Z. kawagoii and Z. shuanglongensis in Taiwan. This is congruent with Bomblies and Weigel's [3] autoimmunity hypothesis for the between-species incompatibility, and the adaptive divergent responses to the same stress support the mutation-order speciation sympatrically within an island. 


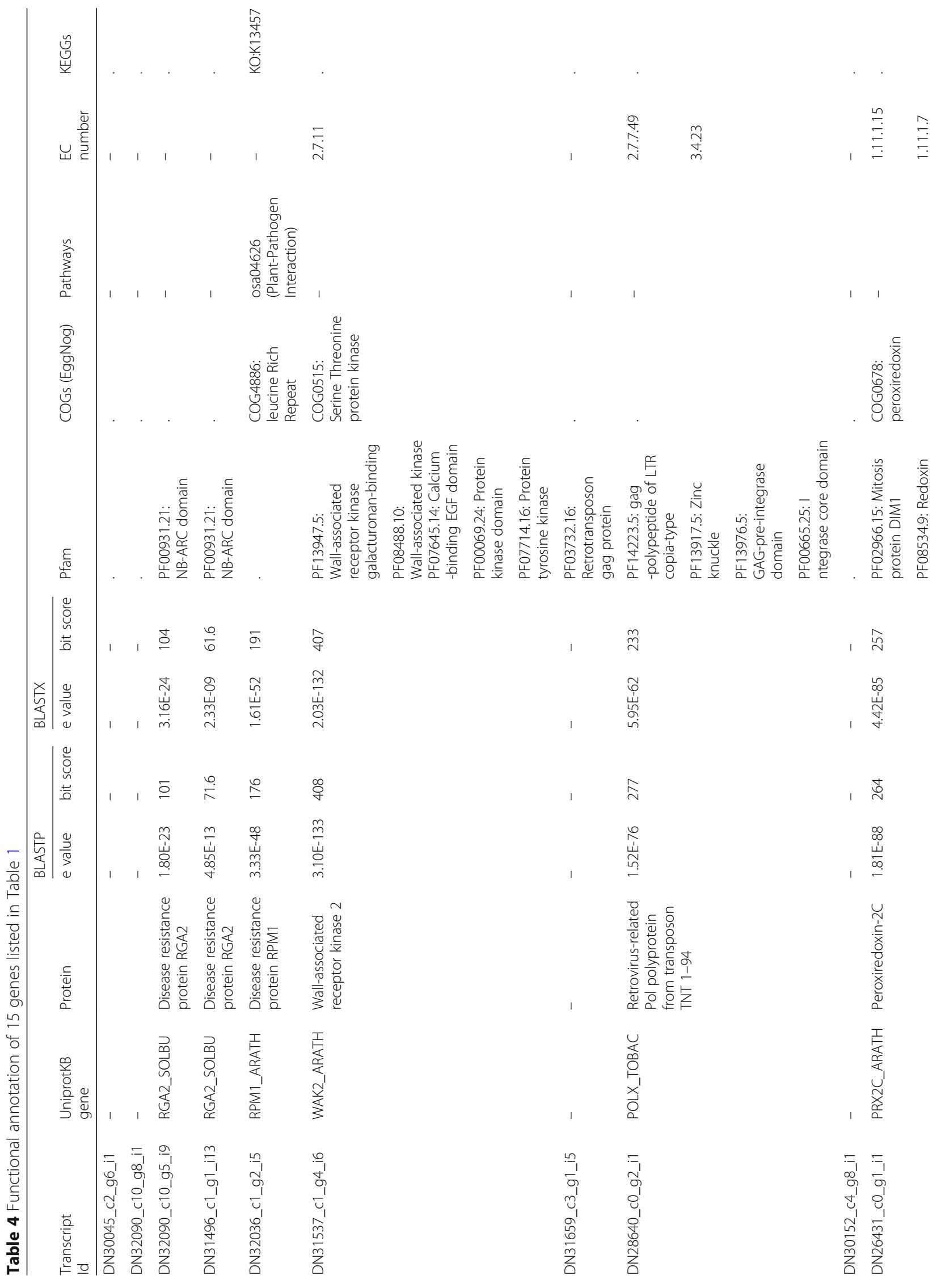




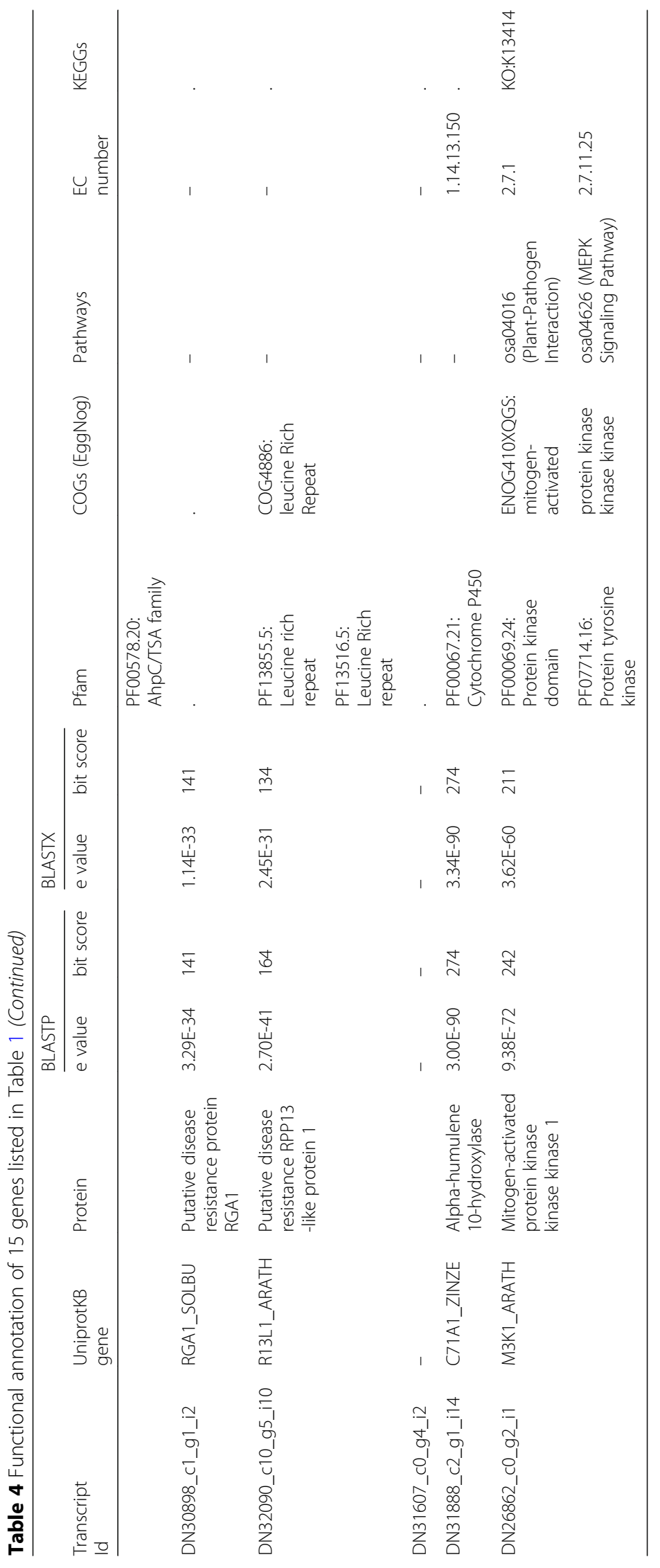




\section{Conclusions}

In conclusion, Z. kawagoii and Z. shuanglongensis is recently diverged and is most probably sympatrically speciated underlying the adaptive divergence of stress-resistance DEGs. Since lack of obvious habitat heterogeneity between species, we suggested mutation-order speciation, a mechanism of fixation of equally advantageous mutations in different populations/incipient species in the face of the same environmental pressures, might explain the adaptive divergence of these two sympatric island species. These adaptiv e-divergence mechanisms are based on the expressional plasticity of incipient speciation to expand adaptabilities of incipient species. Although the speciation mechanism and origination hypothesis need more studies to validate, differential responses to the same stress in genetic variation and expression have explained how sympatric incipient species diverged when suffering identical environmental pressures.

\section{Methods}

\section{Plant material preparation, RNA extraction, and} transcriptome sequencing

Mature plants of Z. kawagoii and Z. shuanglongensis are domesticated for more than a month in the greenhouse at the National Taiwan Normal University after collection from Miaoli Nanzhuang (N24'34'24.1", E121 02'31.7") and Jenlun Forest Road (N25 02'0.6", E121 33'50.8'). Before RNA extraction, the roots of samples were irrigated with $1 \mathrm{M} \mathrm{NaCl}$ for saline stress for $1 \mathrm{~h}$, and then the fresh leaves were collected for RNA extraction immediately using an improved cetyltrimethylammonium bromide (CTAB) method. RNA integrity was checked through $1 \%$ agarose gel electrophoresis, and then the mRNA was purified using poly-T oligo-attached beads. The cDNA libraries of both species were constructed from qualified RNA following manufacture's protocol (Illumina Inc. San Diego, CA, USA). Library quality was validated on Agilent 2100 Bio-analyzer (Agilent Technology, CA, USA) and real-time PCR system. The paired-end sequencing was then performed by Illumina HiSeq platform. To elucidate the within-species variation of both Z. kawagoii and $Z$. shuanglongensis, we adopted three chloroplast regions for studying the level of intra-specific variation, including trn $\mathrm{H}-p s b \mathrm{~A}$ intergenic spacer (forward primer: 5'-GTT ATG CAT GAA CGT AAT GCT C-3', reverse primer: 5'-CGC GCA TGG TGG ATT CAC AAT C-3'), rpl16 intron (forward primer: 5'-CGA AAT CGG TAG ACG CTA CG-3', reverse primer: 5' - ATT TGA ACT GGT GAC ACG AG-3'), and trnL-trnF intergenic spacer (forward primer: 5' - GCT ATG CTT AGT GTG TGA CTC GTT A-3',reverse primer: 5'-CTT CCT CTA TGT TGT TTA CG-3'). We sampled nine Z. kawagoii populations and three Z. shuanglongensis populations (Table 1) for amplification and sequencing using BigDye terminator kit (Applied Biosystems). The divergence index including $F_{\mathrm{ST}}$ and average pairwise $\pi_{X Y}$ were adopted to elucidate the intraspecific diversity. The concatenated sequences were deposited on GenBank (accession number: MH758531-MH 758541), and the aligned file was also deposited in Mendeley (https://data.mendeley.com/datasets/fky8hszf8n/1).

\section{Bioinformatics analysis}

The modular tool MultiQC [64] was used for evaluating read quality. With the FastQC for quality control, the reads contaminated with adapter sequences, containing $\mathrm{N}$ bases accounting for $>5 \%$ of the total read length, or low-quality sequences with Phred score $<28$ were removed using Trimmomatic [65]. Trinity ver. 2.3.2 [66] was used to de novo assembly with a setting of minimum contig length $150 \mathrm{bps}$ and the CD-HIT-EST [67] was used for removing redundant transcripts to obtain more specific unigenes with a $95 \%$ sequence identity threshold. The filtered reads data were deposited in the NCBI Sequence Read Archive (SRA) with the Bioproject accession number PRJNA437070. The backbone reference was de-novo assembled using reads from both species by bowtie2 ver. 2.3.1 [38] and the read count quantification was performed by RSEM ver. 1.2.31 [68]. Relative expression level was calculated by the FPKM using RSEM ver. 1.2.31 [68]. Differential gene expression was then estimated by edgeR ver. 3.5 [69] with a criterion of $\log _{2} \mathrm{FC}>1$ with $P<0.05$. We cross-compared the significant differences of FPKM $(P<0.05)$ and $\log _{2}$ FC $>1$ to confirm the differential expression.

We used the Transdecoder ver. 3.0.1 [https://transdecoder.github.io/] to parse coding region of all transcripts and retrieve protein sequences. Trinotate ver. 3.0.2 [https://trinotate.github.io/] was further used for functional annotation to processing the blastx and blastp homology search in UniProtKB/Swiss-Prot database. We also used the protein families (Pfam) domain database to identity protein domain in predicted and annotated proteins. Clusters of Orthologous Groups (COGs), Gene Ontology (GO), Kyoto Encyclopedia of Genes and Genomes (KEGG) functional and pathway annotation was also conducted. The GO enrichment analysis for gene set was further performed to compare the differences of functional expression groups between two species.

SNPs of homologous transcripts in both species were identified by samtools [70] and bcftools [71]. The SNPs data were saved as the Variant Call Format (VCF) file in which only the SNPs with quality $>30$ were retained. The snpEff [72] and SnpSift [73] were used for SNP annotation and retrieve useful annotated variants, respectively. In the snpEff analysis, functional classes (missense, nonsense, and synonymous variants) and variant regions (3'- and 5'-UTR, intergenic, exonic, and splice-site regions) were classified. 


\section{Testing the adaptive-divergence and plastic-divergence hypotheses}

After the SNP calling, the ratio of missense mutation rate to silent mutation rate of each gene (hereafter named " $\mathrm{Ka} / \mathrm{Ks}$ ") was estimated by assuming two fold nonsynonymous sites more than synonymous sites of coding sequences. Although this is a rough estimation, the estimated $\mathrm{Ka} / \mathrm{Ks}$ ratio is better than direct estimating the proportion of nonsynonymous and synonymous variants (i.e. pa/ps) for the inference of positive selection $(K a / K s>1)$. Among all transcripts with exonic SNPs, we compared the proportion of DEGs to the number of no-expressional-difference genes between those of $K a / K s>1$ and $K a / K s \leq 1$. Since the differential gene expression between species indicates divergent physiological responses to the environment, the positive selection on DEGs indicates the adaptive divergence. In contrast, the DEGs with $K a / K s \leq 1$ indicate the expressional divergence is not genetically based but an acclimated response, i.e. plastic divergence.

\section{Additional files}

Additional file 1: Sequencing depth of coverage across pseudoreference genome. (DOCX $653 \mathrm{~kb}$ )

\begin{abstract}
Abbreviations
BP: Biological Process of the GO term; CC: Cell Component of the GO term; COG: Clusters of Orthologous Groups; cpDNA: Chloroplast DNA;

CTAB: Cetyltrimethylammonium bromide; DEG: Differential expression genes; ENM: Ecological niche modeling; FPKM: The Fragment Per Kilobase of

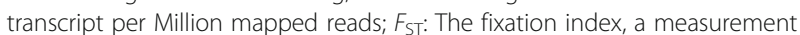
of population differentiation; GO: Gene Ontology; K: The average variants (or fixed differences) per site; Ka: Nonsynonymous mutation rate; KEGG: Kyoto encyclopedia of genes and genomes; Ks: Synonymous mutation rate; $\log _{2}$ FC: $\log _{2}$ fold-change; MF: Molecular Function of the GO term; Mya: Million years ago; Pfam: The protein families; PSGs: Positively selected genes; Q20: The sequencing quality score of 20 (an error rate of 1 in 100, with a corresponding call accuracy of 99\%); QTLs: Quantitative trait loci; RNAseq: RNA sequencing or the transcriptome sequencing; siRNA: small interfering RNAs; SNPs: single nucleotide polymorphisms; SRA: Sequence Read Archive; T: The divergent time; TMRCA: The time to the most recent common ancestor; UTR: Untranslated region; VCF: The Variant Call Format; $\mu$ : Mutation rate; $\pi$ : Nucleotide diversity estimated by pairwise differences; $\pi_{x y}$ : The average nucleotide diversity estimated by $\pi$
\end{abstract}

\section{Acknowledgements}

The authors thank Dawn Schmidt for English editing.

\section{Funding}

This research was financially supported by the Ministry of Science and Technology in Taiwan (MOST 105-2628-B-003 -002 -MY3) and was also subsidized by the National Taiwan Normal University (NTNU), Taiwan.

\section{Availability of data and materials}

The chloroplast DNA sequences were deposited in the NCBI GenBank (accession number: MH758531-MH758541). The filtered reads data were deposited in the NCBI Sequence Read Archive (SRA) with the Bioproject accession number PRJNA437070. The assembly and expression profiles in this study were deposited in the Mendeley Data (https://data.mendeley.com; https://doi.org/10.17632/mxfgjzyxm9.1).

\section{Authors' contributions}

$\mathrm{PL}$ and $\mathrm{YL}$ conceived this study. $\mathrm{BH}, \mathrm{HL}$ and $\mathrm{ML}$ collected materials. $\mathrm{BH}, \mathrm{CH}$, $\mathrm{HL}$ and $\mathrm{ML}$ conducted experiments. $\mathrm{BH}$ and $\mathrm{PL}$ analysed the data. $\mathrm{PL}$ wrote the paper. $\mathrm{YL}, \mathrm{CH}$ and $\mathrm{BH}$ critically reviewed the manuscript. All authors participated in the discussion and read and approved the final manuscript.

\section{Competing interest}

The authors declare that they have no competing interest.

\section{Ethics approval and consent to participate}

Not applicable.

\section{Consent for publication}

Not applicable.

\section{Publisher's Note}

Springer Nature remains neutral with regard to jurisdictional claims in published maps and institutional affiliations.

\section{Author details}

${ }^{1}$ School of Life Science, National Taiwan Normal University, No. 88, Sec. 4, Ting-Chow Rd., Wenshan Dist, Taipei 11677, Taiwan. ²Department of Forestry, National Chung-Hsing University, No. 250, Kuo Kuang Rd, Taichung 402, Taiwan.

Received: 14 June 2018 Accepted: 14 September 2018

Published online: 21 September 2018

\section{References}

1. Michel AP, Sim S, Powell THQ, Taylor MS, Nosil P, Feder JL. Widespread genomic divergence during sympatric speciation. Proc Natl Acad Sci. 2010; 107(21):9724-9.

2. Lexer C, Fay MF. Adaptation to environmental stress: a rare or frequent driver of speciation? J Evolution Biol. 2005;18(4):893-900.

3. Bomblies K, Weigel D. Hybrid necrosis: autoimmunity as a potential geneflow barrier in plant species. Nat Rev Genet. 2007;8:382-93.

4. Greenberg AJ, Moran JR, Coyne JA, Wu C-I. Ecological adaptation during incipient speciation revealed by precise gene replacement. Science. 2003; 302(5651):1754-7.

5. Schluter D. Evidence for ecological speciation and its alternative. Science. 2009;323(5915):737-41.

6. Flatscher R, Frajman B, Schönswetter P, Paun O. Environmental heterogeneity and phenotypic divergence: can heritable epigenetic variation aid speciation? Genetics Research International. 2012;2012:698421.

7. Stanton ML, Roy BA, Thiede DA. Evolution in stressful environments. I. Phenotypic variability, phenotypic selection, and response to selection in five distinct environmental stresses. Evolution. 2000;54(1):93-111.

8. Nosil P. Flaxman SM. Conditions for mutation-order speciation. Proc R Soc B Biol Sci. 2011;278(1704):399-407.

9. Bank C, Bürger R, Hermisson J. The limits to parapatric speciation: Dobzhansky-Muller incompatibilities in a continent-island model. Genetics. 2012;191(3):845-63.

10. Papadopulos AST, Baker WJ, Crayn D, Butlin RK, Kynast RG, Hutton I, Savolainen V. Speciation with gene flow on Lord Howe Island. Proc Natl Acad Sci. 2011;108(32):13188-93.

11. Savolainen V, Anstett M-C, Lexer C, Hutton I, Clarkson JJ, Norup MV, Powell MP, Springate D, Salamin N, Baker WJ. Sympatric speciation in palms on an oceanic island. Nature. 2006:441:210-3.

12. Babik W, Butlin RK, Baker WJ, Papadopulos AST, Boulesteix M, Anstett M-C, Lexer C, Hutton IAN, Savolainen V. How sympatric is speciation in the Howea palms of Lord Howe Island? Mol Ecol. 2009;18(17):3629-38.

13. Li J-W, Yeung CKL, Tsai P-W, Lin R-C, Yeh C-F, Yao C-T, Han L, Hung LM, Ding $P$, Wang $Q$, et al. Rejecting strictly allopatric speciation on a continental island: prolonged postdivergence gene flow between Taiwan (Leucodioptron taewanus, Passeriformes Timaliidae) and Chinese (L. canorum canorum) hwameis. Mol Ecol. 2010;19(3):494-507.

14. Huang B-H, Huang C-W, Huang C-L, Liao P-C. Continuation of the genetic divergence of ecological speciation by spatial environmental heterogeneity in island endemic plants. Sci Rep. 2017;7(1):5465.

15. Thorpe RS, Surget-Groba Y, Johansson H. Genetic tests for ecological and allopatric speciation in anoles on an island archipelago. PLoS Genet. 2010;6(4):e1000929. 
16. Lee $Y-H$, Lin C-P. Pleistocene speciation with and without gene flow in Euphaea damselflies of subtropical and tropical east Asian islands. Mol Ecol. 2012;21(15):3739-56.

17. Tseng S-P, Wang C-J, Li S-H, Lin S-M. Within-island speciation with an exceptional case of distinct separation between two sibling lizard species divided by a narrow stream. Mol Phylogenet Evol. 2015;90:164-75.

18. Tseng S-P, Li S-H, Hsieh C-H, Wang H-Y, Lin S-M. Influence of gene flow on divergence dating - implications for the speciation history of Takydromus grass lizards. Mol Ecol. 2014;23(19):4770-84.

19. Lin S-M, Chen CA, Lue K-Y. Molecular phylogeny and biogeography of the grass lizards genus Takydromus (Reptilia: Lacertidae) of East Asia. Mol Phylogenet Evol. 2002;22(2):276-88.

20. Tseng H-Y, Huang W-S, Jeng M-L, Villanueva RJT, Nuñeza OM, Lin C-P. Complex inter-island colonization and peripatric founder speciation promote diversification of flightless Pachyrhynchus weevils in the TaiwanLuzon volcanic belt. J Biogeogr. 2018;45(1):89-100.

21. Nurainas $N$, Arbain D. A new species and a new record of Zingiberaceae from Sumatra Indonesia. Taiwania. 2017;62(3):294-8.

22. Chaveerach A, Mokkamul P, Sudmoon R, Tanee T. A new species of Zingiber (Zingiberaceae) from northern Thailand. Taiwania. 2007;52(2):159-63.

23. Yeh C-L, Chung S-W, Kuo Y-W, Hsu T-C, Leou C-S, Hong S-J, Yeh C-R. A new species of Zingiber (Zingiberaceae) from Taiwan, China, based on morphological and molecular data. J Syst Evol. 2012;50(2):163-9.

24. Bai L, Leong-Škorničková J, Li D, Xia N: Taxonomic studies on Zingiber (Zingiberaceae) in China IV: Z. pauciflorum, a new species from Yunnan. Nordic Journal of Botany 2017:(in press).

25. Govaerts R. Nomenclatural notes in Asian Zingiberaceae. Taiwania. 2016;61(3):269-70.

26. Lin Y-C. Taxonomic study of Zingiber mill. (Zingiberaceae) in Taiwan. M.S Thesis. Taichung, Taiwan: National Chung-Hsing University; 2017.

27. Bai L, Leong-ŠKKorniČKovÁ J, Xia N-H, Ye Y-S. Taxonomic studies on Zingiber (Zingiberaceae) in China III: Z. ventricosum, a new species from Yunnan, and notes on three closely related species. Phytotaxa. 2016;261(2):18.

28. Liu S-C, Lu C-T, Wang J-C. Reticulate hybridization of Alpinia (Zingiberaceae) in Taiwan. J Plant Res. 2009;122(3):305-16.

29. Valderrama E, Richardson JE, Kidner CA, Madriñán S, Stone GN. Transcriptome mining for phylogenetic markers in a recently radiated genus of tropical plants (Renealmia L.f., Zingiberaceae). Mol Phylogenet Evol. 2018;119:13-24.

30. Mani GS, Clarke BC. Mutational order: a major stochastic process in evolution. Proceedings of the Royal Society of London B Biological Sciences. 1990;240(1297):29

31. Pfennig DW, Wund MA, Snell-Rood EC, Cruickshank T, Schlichting CD, Moczek AP. Phenotypic plasticity's impacts on diversification and speciation. Trends Ecol Evol. 2010;25(8):459-67.

32. Grenier S, Barre P, Litrico I. Phenotypic plasticity and selection: nonexclusive mechanisms of adaptation. Scientifica. 2016;2016:7021701.

33. Nonaka E, Svanbäck R, Thibert-Plante X, Englund G, Brännström A Mechanisms by which phenotypic plasticity affects adaptive divergence and ecological speciation. Am Nat. 2015;186(5):E126-43.

34. Thibert-Plante $X$, Hendry AP. The consequences of phenotypic plasticity for ecological speciation. J Evolution Biol. 2011;24(2):326-42.

35. Asensi-Fabado M-A, Amtmann A, Perrella G. Plant responses to abiotic stress: the chromatin context of transcriptional regulation. Biochimica et Biophysica Acta (BBA) - Gene Regulatory Mechanisms. 2017;1860(1):106-22.

36. Ohama N, Sato H, Shinozaki K, Yamaguchi-Shinozaki K. Transcriptional regulatory network of plant heat stress response. Trends Plant Sci. 2017;22(1):53-65.

37. Edwards S, Beerli P. Perspective: gene divergence, population divergence, and the variation in coalescence time in phylogeographic studies. Evolution. 2000;54(6):1839-54.

38. Langdon WB. Performance of genetic programming optimised Bowtie2 on genome comparison and analytic testing (GCAT) benchmarks. BioData Mining. 2015;8(1):1.

39. Ossowski S, Schneeberger K, Lucas-Lledó Jl, Warthmann N, Clark RM, Shaw RG, Weigel D, Lynch M. The rate and molecular spectrum of spontaneous mutations in Arabidopsis thaliana. Science. 2010;327(5961):92-4.

40. Nei $M$, Gojobori T. Simple methods for estimating the numbers of synonymous and nonsynonymous nucleotide substitutions. Mol Biol Evol. 1986;3(5):418-26.

41. Lockwood BL, Sanders JG, Somero GN. Transcriptomic responses to heat stress in invasive and native blue mussels (genus <em>Mytilus</em>) molecular correlates of invasive success. J Exp Biol. 2010;213(20):3548-58.
42. Voelckel C, Gruenheit N, Lockhart P. Evolutionary transcriptomics and proteomics: insight into plant adaptation. Trends Plant Sci. 2017;22(6):462-71.

43. Nekrutenko A, Makova KD, Li W-H. The $K_{A} / K_{S}$ ratio test for assessing the protein-coding potential of genomic regions: an empirical and simulation study. Genome Res. 2002;12(1):198-202.

44. Capy P, Gasperi G, Biémont C, Bazin C. Stress and transposable elements: co-evolution or useful parasites? Heredity. 2000;85:101-6.

45. Grandbastien M-A. Activation of plant retrotransposons under stress conditions. Trends Plant Sci. 1998;3(5):181-7.

46. Wessler SR. Plant retrotransposons: turned on by stress. Curr Biol. 1996;6(8):959-61.

47. Matsunaga W, Ohama N, Tanabe N, Masuta Y, Masuda S, Mitani N, Yamaguchi-Shinozaki K, Ma JF, Kato A, Ito H. A small RNA mediated regulation of a stress-activated retrotransposon and the tissue specific transposition during the reproductive period in Arabidopsis. Front Plant Sci. 2015:6:48.

48. Traylor-Knowles N, Rose NH, Sheets EA, Palumbi SR. Early transcriptional responses during heat stress in the coral Acropora hyacinthus. Biol Bull. 2017;232(2):91-100.

49. Ramallo E, Kalendar R, Schulman AH, Martínez-Izquierdo JA. Reme1, a Copia retrotransposon in melon, is transcriptionally induced by UV light. Plant Mol Biol. 2007;66(1):137.

50. Pearson GA, Hoarau G, Lago-Leston A, Coyer JA, Kube M, Reinhardt R, Henckel K, Serrão ETA, Corre E, Olsen JL. An expressed sequence tag analysis of the intertidal brown seaweeds Fucus serratus (L.) and $F$. vesiculosus (L.) (Heterokontophyta, Phaeophyceae) in response to abiotic stressors. Mar Biotechnol. 2010;12(2):195-213.

51. Shukla $Y$, Singh M. Cancer preventive properties of ginger: a brief review. Food Chem Toxicol. 2007;45(5):683-90.

52. Haque MA, Jantan I, Arshad L, Bukhari SNA. Exploring the immunomodulatory and anticancer properties of zerumbone. Food Funct. 2017:8(10):3410-31.

53. Kalantari K, Moniri M, Boroumand Moghaddam A, Abdul Rahim R, Bin Ariff A, Izadiyan Z, Mohamad R. A review of the biomedical applications of zerumbone and the techniques for its extraction from ginger rhizomes. Molecules. 2017;22(10):1645.

54. Teng LS. Extensional collapse of the northern Taiwan mountain belt. Geology. 1996;24(10):949-52.

55. Chi W-R, Namson J, Suppe J. Stratigraphic record of plate interactions in the coastal range of eastern Taiwan. Mem Geol Soc China. 1981;4:155-94.

56. Wang CS, Huang CP, Ke LY, Chien WJ, Hsu SK, Shyu CT, Cheng WB, Lee CS, Teng LS. Formation of the Taiwan island as a solitary wave along the Eurasian continental plate margin: magnetic and seismological evidence. Terr Atmos Ocean Sci. 2002;13(3):339-54.

57. Crispo E. Modifying effects of phenotypic plasticity on interactions among natural selection, adaptation and gene flow. J Evol Biol. 2008;21(6):1460-9.

58. Pavey SA, Collin H, Nosil P, Rogers SM. The role of gene expression in ecological speciation. Ann N Y Acad Sci. 2010;1206(1):110-29.

59. Agrawal AA. Phenotypic plasticity in the interactions and evolution of species. Science. 2001;294(5541):321-6.

60. Becklin KM, Anderson JT, Gerhart LM, Wadgymar SM, Wessinger CA, Ward JK. Examining plant physiological responses to climate change through an evolutionary lens. Plant Physiol. 2016;172(2):635-49.

61. O'Hara NB, Rest JS, Franks SJ. Increased susceptibility to fungal disease accompanies adaptation to drought in Brassica rapa. Evolution. 2016;70(1):241-8.

62. Lexer C, Welch ME, Durphy JL, Rieseberg LH. Natural selection for salt tolerance quantitative trait loci (QTLs) in wild sunflower hybrids: implications for the origin of Helianthus paradoxus, a diploid hybrid species. Mol Ecol. 2003;12(5):1225-35

63. Lexer C, Lai Z, Rieseberg LH. Candidate gene polymorphisms associated with salt tolerance in wild sunflower hybrids: implications for the origin of Helianthus paradoxus, a diploid hybrid species. New Phytol. 2004;161(1):225-33.

64. Ewels P, Magnusson M, Lundin S, Käller M. MultiQC: summarize analysis results for multiple tools and samples in a single report. Bioinformatics. 2016;32(19):3047-8

65. Bolger AM, Lohse M, Usadel B. Trimmomatic: a flexible trimmer for Illumina sequence data. Bioinformatics. 2014;30(15):2114-20.

66. Grabherr MG, Haas BJ, Yassour M, Levin JZ, Thompson DA, Amit I, Adiconis X, Fan L, Raychowdhury R, Zeng Q, et al. Trinity: reconstructing a full-length transcriptome without a genome from RNA-Seq data. Nat Biotechnol. 2011;29(7):644-52. 
67. Li W, Godzik A. Cd-hit: a fast program for clustering and comparing large sets of protein or nucleotide sequences. Bioinformatics. 2006;22(13):1658-9.

68. Li B, Dewey CN. RSEM: accurate transcript quantification from RNA-Seq data with or without a reference genome. BMC Bioinformatics. 2011;12(1):323.

69. Robinson MD, McCarthy DJ, Smyth GK. edgeR: a Bioconductor package for differential expression analysis of digital gene expression data. Bioinformatics. 2010;26(1):139-40.

70. Li H, Handsaker B, Wysoker A, Fennell T, Ruan J, Homer N, Marth G, Abecasis G, Durbin R. The sequence alignment/map format and SAMtools. Bioinformatics. 2009;25(16):2078-9.

71. Narasimhan V, Danecek P, Scally A, Xue Y, Tyler-Smith C, Durbin R. BCFtools/ RoH: a hidden Markov model approach for detecting autozygosity from next-generation sequencing data. Bioinformatics. 2016;32(11):1749-51.

72. Cingolani P, Platts A, Wang LL, Coon M, Nguyen T, Wang L, Land SJ, Lu X, Ruden DM. A program for annotating and predicting the effects of single nucleotide polymorphisms, SnpEff. Fly. 2012;6(2):80-92.

73. Ruden D, Cingolani P, Patel V, Coon M, Nguyen T, Land S, Lu X. Using Drosophila melanogaster as a Model for Genotoxic Chemical Mutational Studies with a New Program, SnpSift. Front Genet. 2012;3:35.

Ready to submit your research? Choose BMC and benefit from:

- fast, convenient online submission

- thorough peer review by experienced researchers in your field

- rapid publication on acceptance

- support for research data, including large and complex data types

- gold Open Access which fosters wider collaboration and increased citations

- maximum visibility for your research: over $100 \mathrm{M}$ website views per year

At $\mathrm{BMC}$, research is always in progress.

Learn more biomedcentral.com/submissions 